\title{
La simplification du contrôle des cultures de chanvre industriel est possible
}

\author{
The simplification of the industrial hemp \\ crops control is possible
}

\section{Gilbert FOURNIER $^{(1) *}$, Jocelyne BAUSSET ${ }^{(1)}$, Olivier BEHEREC ${ }^{(2)}$, Maud DESVALS ${ }^{(2)}$, Sylvestre BERTUCELLI ${ }^{(2)}$}

(1) Laboratoire de Pharmacognosie, UMR 8076, CNRS (BioCIS), Faculté de Pharmacie, 5, rue J.-B. Clément F-92296 Châtenay-Malabry Cedex

(2) Fédération Nationale des Producteurs de Chanvre (FNPC), 20, rue Paul Ligneul - F-72000 Le Mans

*Auteur à qui adresser la correspondance : Professeur Gilbert FOURNIER, Laboratoire de Pharmacognosie, UMR
8076, CNRS (BioCIS), Faculté de Pharmacie, 5, rue J.-B. Clément - F-92296 Châtenay-Malabry Cedex
Tél. : 0146835597 - Fax : 0146835710 - E-mail : gilbert.fournier@u-psud.fr

(Reçu le 22 juin 2007 ; accepté après modification le 3 septembre 2007)

\section{$\boldsymbol{R E ́} \boldsymbol{E} U \boldsymbol{M E ́}$}

Chaque année sont effectués plus de 800 contrôles du chanvre industriel cultivé en Europe sur près de 16000 hectares. Il s'agit de déterminer la teneur en $\Delta^{y}-T H C$, substance psychotrope spécifique du chanvre. La norme actuelle ( $\Delta^{9}-$ $T H C \leq 0,20 \%)$ a été officialisée en même temps qu'ont été modifiées les procédures standardisées de prélèvement et d'analyse des échantillons. Depuis 2000, la procédure actuellement en vigueur remplace une procédure contraignante et fastidieuse qui nécessitait des prélèvements volumineux à réaliser sur une période très courte (durant la fin de la floraison). Cependant, la mise en auvre de cette procédure a induit un accroissement artificiel des valeurs moyennes mesurées et une variabilité accrue de celles-ci. Dans ce travail il est tout d'abord montré qu'une corrélation forte $\left(R^{2}>0,9\right)$ existe entre la teneur en $\Delta^{9}-T H C$ et le rapport $\alpha=\Delta^{9}-T H C / C B D$ pour l'ensemble des variétés (13) ou des ressources génétiques (15) testées. Ceci montre que $\alpha$ peut donc remplacer la simple détermination du $\Delta^{9}-T H C$. Il est ensuite montré que contrairement à la seule détermination du $\Delta^{y}-T H C, \alpha$ est constant quelle que soit la partie prélevée et l'époque du prélèvement. Un échantillon constitué de 100

\section{SUMMARY}

Each year; more than 800 controls are carried out on industrial hemp cultivated throughout Europe over about 40,000 acres. They aim at measuring the $\triangle-9-T H C$ content, which is the psychotropic substance specific to hemp. The present standard value $(\Delta-9-T H C<0.2 \%)$ has been adopted and sample analysis protocols have been modified. The new method came into force in 2000 and substitutive for the previously restrictive and tedious method which entailed the taking of numerous samples over a very short time period (at the end of the blossoming). However, the implementation of this new method has resulted in an increase of the mean $\Delta$ 9-THC content and of their variability.

In this study a close correlation $\left(R^{2}>0.9\right)$ between $\Delta-9-T H C$ content and $\triangle-9-T H C / C B D$ ratio $\alpha$ was found for the all set of varieties (13) and germplasm (15) tested. $\alpha$ can then substitute to the only determination of $\triangle-9-T H C$ content. $\alpha$ also revealed to be constant what ever part of the plant was sampled or whenever it would be sampled. A sample composed of 100 plants guaranty enough reliability of the results.

The proposition was to sample one leaf of 100 plants at any 
plantes en mélange assure une fiabilité suffisante du résultat. La proposition est donc de prélever une feuille sur 100 plantes à n'importe quel moment du cycle végétatif et de déterminer $\alpha=\triangle^{\prime}-T H C / C B D$. Un $\alpha \leq 0,20$ assure une teneur en $\triangle^{9}$-THC conforme à la norme communautaire en vigueur $(\leq 0,20 \%)$ applicable aux cultures de chanvre industriel.

\section{MOTS CLÉS}

Chanvre industriel, Cannabis sativa, delta-9-tétrahydrocannabinol, cannabidiol, réglementation.

\section{Introduction}

Les propriétés psychotropes du chanvre (Cannabis sativa L.) sont classiquement dues au $\Delta$-9-tétrahydrocannabinol $\left(\Delta^{9}\right.$-THC), substance spécifique de cette plante.

Dans l'Union Européenne, le contrôle des cultures de chanvre industriel est actuellement effectué à partir d'un échantillon standardisé et consiste à vérifier que la teneur en $\Delta^{\circ}$-THC de cet échantillon ne dépasse pas $0,20 \%$. Il a ainsi été réalisé 813 analyses en 2004, 887 en 2005 et 829 en 2006.

Historiquement créée en 1989 (1), et reprise en 1990 par le Code de la Santé Publique français (2), la procédure était utilisée pour déterminer la teneur en $\Delta^{9}$ THC des variétés lors de leur inscription au Catalogue des Variétés, et était de fait appliquée sur un nombre restreint d'échantillons regroupés géographiquement. Une décision Communautaire a rendu obligatoire le contrôle de cultures de chanvre industriel, en utilisant la procédure en vigueur à cette époque. Il est alors apparu que cette procédure posait de graves problèmes logistiques compte tenu des volumes importants de matières à transporter dans des délais très courts et sur une aire géographique à l'échelle de chaque pays. Pour résoudre ces problèmes, cette procédure initiale (notée PI dans la suite du document) a évolué en 2000 pour conduire à deux procédures différentes, l'une (simplifiée) (notée A) s'appliquant au contrôle en culture et l'autre (notée $\mathrm{B}$ ) réservée à l'inscription des variétés (3-4). Le tableau I résume ces différentes procédures et leurs champs d'application. Cette modification de la procédure d'échantillonnage s'est accompagnée, la même année (2000), d'un changement de la norme pour la teneur maximale en $\Delta^{9}$-THC, qui est passée de $0,30 \%$ à $0,20 \%(5)$. Une étude critique de ces deux procédures d'échantillonnage $(\mathrm{A}$ et $\mathrm{B}$ ) a été réalisée (6). Elle se base sur le fait qu'il est désormais bien admis que la teneur en $\Delta^{9}$-THC varie au sein d'une même plante, d'une part en fonction de la partie prélevée (gradient croissant du bas vers le haut dans les par- stage of the vegetative cycle and to determine the $\alpha=\Delta-9$ THC/CBD. An $\alpha \leq 0.20$ guaranties a $\triangle-9-T H C$ content conform to Community standard value $(\leq 0.20)$ applicable to industrial hemp crops.

\section{KEY-WORDS}

Industrial hemp, Cannabis sativa, delta-9-tetrahydrocannabinol, cannabidiol, regulation.

ties vertes de la plante) et, d'autre part, au cours du développement temporel de la plante. Ces variations conduisent, bien évidemment, à des résultats différents et hétérogènes selon la procédure mise en œuvre. Par ailleurs, selon les proportions relatives de $\Delta^{y}$-THC, de cannabidiol (CBD), cannabinoïde non psychotrope et biogénétiquement proche du $\Delta^{y}$-THC, et de cannabigérol (CBG), cannabinö̈de également non psychotrope précurseur des précédents, plusieurs chimiotypes ont été décrits : «Drogue » $\left(\Delta^{9}-\mathrm{THC}>\mathrm{CBD}\right)$, « Drogue intermédiaire » (teneurs en $\Delta^{9}$-THC et en CBD voisines), « Fibres $»\left(\Delta^{9}\right.$-THC $<<$ CBD) $(7-16)$, « Fibres de Seconde Génération» ( $\Delta^{\prime}$-THC absent, CBD présent à l'état de trace, CBG majoritaire) (17) et " Fibres de Troisième Génération » $\left(\Delta^{9}-\mathrm{THC}, \mathrm{CBD}\right.$ et $\mathrm{CBG}$ absents) (18).

Les modifications appliquées depuis 2000 uniquement dans un souci logistique ont notamment eu pour conséquence, avec la procédure A, d'augmenter les valeurs mesurées, en prenant un échantillon plus court et plus proche de la sommité, et également d'augmenter leur variabilité, en prenant un échantillon constitué à partir de beaucoup moins de plantes. Un tel échantillon est de fait peu représentatif de la variété et la procédure moins fiable que celle utilisée pour l'inscription.

Nous avons récemment montré l'intérêt de la prise en considération du rapport $\alpha=\Delta^{9}$-THC / CBD dans le contrôle des cultures de chanvre industriel (19). Cette étude avait été principalement réalisée à partir des résultats des dosages des cannabinoïdes obtenus avec une variété autorisée à la culture en Europe : Fédora 17, et une ancienne variété roumaine aujourd'hui disparue des catalogues : Secueni, dont la teneur en $\Delta^{\prime \prime}$-THC dépasse la norme légale en vigueur dans l'Union Européenne. Lors de cette étude, il avait été constaté que les variations des teneurs en $\Delta^{9}$-THC et en CBD sont parallèles, d'une part selon la partie de la plante prélevée et, d'autre part, selon le stade végétatif des plantes, et ce quelle que soit la variété. Il a donc été montré que le rapport $\alpha=\Delta^{\prime}$-THC / CBD étant, de fait, quasi constant pour une variété donnée, sa prise en 
Tableau I : Selon différents textes réglementaires, rappel des conditions d'échantillonnage à appliquer pour la détermination quantitative du $\Delta^{\prime}-T H C$ des variétés de chanvre.

\begin{tabular}{|c|c|c|c|}
\hline & \multirow[t]{2}{*}{$\begin{array}{c}\text { Règlement (CE) No } \\
1164 / 89 \text { de la } \\
\text { Commission du } 28 \\
\text { avril } 1989 \text { relatif aux } \\
\text { modalités d'aide } \\
\text { concernant le lin } \\
\text { textile et le chanvre (1) } \\
\text { Arrêté du } 22 \text { août } 1990 \\
\text { portant application de } \\
\text { l'article R. } 5181 \text { pour } \\
\text { le cannabis (2) }\end{array}$} & \multicolumn{2}{|c|}{$\begin{array}{c}\text { Règlement (CE) } \mathrm{N}^{\circ} 1177 / 2000 \text { de la } \\
\text { Commission du } 31 \text { mai } 2000 \text { modifiant le } \\
\text { règlement (CEE) n } n^{\circ} 1164 / 89 \text { relatif aux } \\
\text { modalités d'aide concernant le lin textile et le } \\
\text { chanvre (3) } \\
\text { Arrêté du } 24 \text { février } 2004 \text { modifiant l'arrêté du } \\
22 \text { août } 1990 \text { portant application de l'article } \mathrm{R} \text {. } \\
5181 \text { du code de la santé publique pour le } \\
\text { cannabis (4) }\end{array}$} \\
\hline & & Procédure A & Procédure B \\
\hline Champ d'application & $\begin{array}{l}\text { Pour le contrôle des variétés } \\
\text { de cannabis dépourvues de } \\
\text { propriétés stupéfiantes, lors } \\
\text { de l'inscription dans la liste } \\
\text { des variétés éligibles à } \\
\text { l'aide }\end{array}$ & $\begin{array}{l}\text { Pour le contrôle de la } \\
\text { production }\end{array}$ & $\begin{array}{l}\text { Lors de l'inscription dans la } \\
\text { liste des variétés éligibles à } \\
\text { l'aide }\end{array}$ \\
\hline Prélèvements & & & \\
\hline Taille de l'échantillon & $\begin{array}{l}500 \text { plantes } \\
\text { dans une population d'une } \\
\text { variété }\end{array}$ & $\begin{array}{c}50 \text { plantes } \\
\text { dans chaque parcelle }\end{array}$ & $\begin{array}{c}200 \text { plantes } \\
\text { dans chaque parcelle }\end{array}$ \\
\hline Partie à prélever & Toute la plante & $\begin{array}{l}30 \mathrm{~cm} \text { supérieurs contenant } \\
\text { au moins une inflorescence } \\
\text { femelle pour chaque plante } \\
\text { sélectionnée }\end{array}$ & $\begin{array}{l}\text { Tiers supérieur de chaque } \\
\text { plante sélectionnée }\end{array}$ \\
\hline Matériel vẻgétal analysé & $\begin{array}{l}\text { Tiers supérieur débarrassé } \\
\text { des tiges et des graines }\end{array}$ & Matériel végétal prélevé déba & rrassé des tiges et des graines \\
\hline Epoque du prélèvement & fin de la floraison & $\begin{array}{c}\text { Période comprise entre les } \\
20 \text { jours après le début et les } \\
10 \text { jours après la fin de la } \\
\text { floraison }\end{array}$ & $\begin{array}{l}\text { Au cours des } 10 \text { jours } \\
\text { suivant la fin de la floraison }\end{array}$ \\
\hline Teneur max. en $\Delta^{9}$-THC & $\leq 0,30 \%$ & $\leq 0$ & $20 \%$ \\
\hline
\end{tabular}

considération pour le contrôle était beaucoup plus fiable que la seule détermination du $\Delta^{9}$-THC puisqu'il est indépendant de la partie prélevée et de la date du prélèvement.

Afin de faire une nouvelle proposition méthodologique, nous présentons ici les résultats d'expérimentations spécialement mises en œuvre en 2006. Elles ont tout d'abord permis de comparer les résultats de 13 variétés développées par la Fédération Nationale des
Producteurs de Chanvre (FNPC) à ceux de 15 populations de chanvre issues de la collection génétique de ce même organisme. Elles ont ensuite concerné l'étude des variations des teneurs en $\Delta^{9}-\mathrm{THC}$, comparées à celles du rapport $\alpha=\Delta^{\prime}$-THC / CBD, afin d'évaluer le degré de corrélation entre ces deux critères et d'ainsi mieux définir la partie de la plante à prélever, la période pendant laquelle le prélèvement peut être effectué, et la taille de l'échantillon. 


\section{Évaluation d'une relation entre le $\Delta^{9}$-THC et $\alpha=\Delta^{9}$ - $\mathrm{THC} / \mathrm{CBD}$}

\section{Analyse de différentes variétés de chanvre développées par la FNPC}

\section{Méthodologie}

Cette expérimentation a concerné 13 variétés : Epsilon 68, Fédora 17, Fédora 19, Fédrina 74, Félina 32, Félina 34, Férimon, Futura 75, Futura, Juso 14, Santhica 27, Santhica 70 et Uso 31 . Les cultures obtenues étaient représentatives des cultures classiques de chanvre à fibre.

Dans un premier temps, pour l'échantillonnage, nous avons utilisé la procédure initiale (PI), certes lourde à mettre en œuvre en pratique, mais dont les résultats sont classiquement homogènes. Chaque échantillon est ainsi constitué du mélange des tiers supérieurs de 500 plantes prélevées au stade " fin floraison » de la variété, 20 répétitions sont réalisées par variété. Le dosage des cannabinoïdes ( $\Delta^{\prime}$-THC et CBD) est effectué par chromatographie en phase gazeuse selon la procédure officielle (annexe $\mathrm{C}$ du règlement $\mathrm{CE} \mathrm{n}^{\circ} 1177 / 2000 \mathrm{de}$ la Commission du 31 mai 2000) (3). La limite de quantification pour ces cannabinoïdes est de $10^{-5}$.

\section{Résultats et discussion}

Les résultats des dosages des 20 échantillons appartenant à chacune des 13 variétés sont présentés figure 1 qui indique $\alpha=\Delta^{9}$-THC / CBD en fonction du $\Delta^{9}$-THC. Sur l'ensemble de la gamme variétale (variétés françaises et ukrainiennes) développée actuellement par la FNPC et par la Coopérative Centrale des Producteurs de Semences de Chanvre (CCPSC), il existe une corrélation très forte $\left(R^{2}=0,95\right)$ entre la valeur du $\Delta^{9}$-THC et la mesure du rapport $\alpha=\Delta^{\prime}$-THC / CBD dès lors que le prélèvement est fait sur un grand nombre de plantes et concerne leur tiers supérieur. Il apparaît de plus clairement (Figure 1) que la mesure du rapport $\alpha=\Delta^{y}-\mathrm{THC}$ / CBD présente toujours un écart type plus petit que celui relatif au $\Delta^{\prime \prime}$-THC.

\section{Analyse comparative des variétés dévelop- pées par la FNPC et de ressources géné- tiques de chanvre issues de la collection FNPC}

\section{Méthodologie}

Nous avons utilisé ici la même méthodologie que précédemment, en incorporant à l'étude précédente 15 populations de chanvre issues de la collection génétique de la FNPC, notées $\mathrm{P} 01$ à P15.

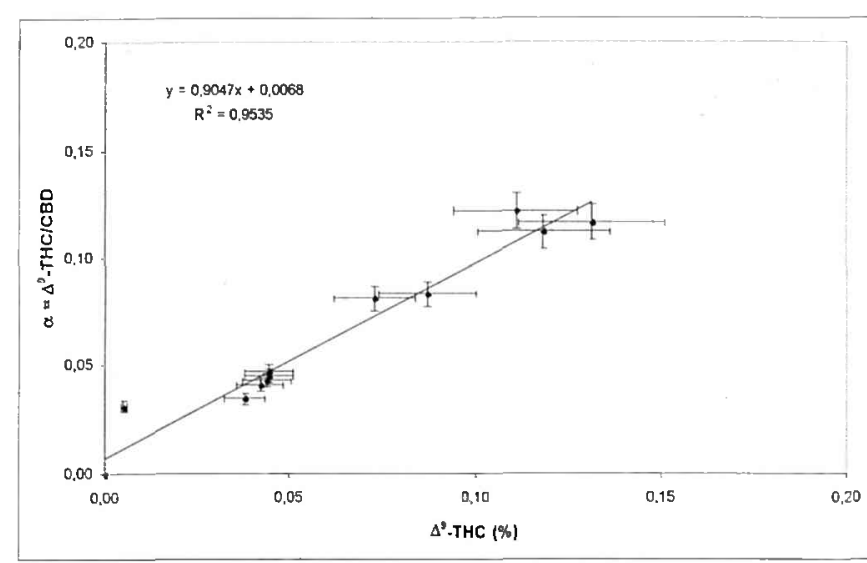

Figure 1 : Relation entre la teneur en $\Delta^{9}-T H C$ et la valeur du rapport $\alpha=\Delta^{9}-T H C / C B D$ (moyennes $+/$ - écart-type, $n=20)$ dans les 13 variétés de chanvre à fibres développées par la FNPC.

\section{Résultats et discussion}

Comme précédemment, les résultats des dosages sont présentés figure 2 qui indique, de la même façon, $\alpha=$ $\Delta^{9}$-THC / CBD en fonction du $\Delta^{9}$-THC.

Cette figure 2 confirme que la corrélation mise en évidence ci-dessus entre la teneur en $\Delta^{9}$-THC et la mesure du rapport $\alpha=\Delta^{9}-\mathrm{THC} / \mathrm{CBD}\left(\mathrm{R}^{2}=0,95\right)$ reste vraie pour l'ensemble des 28 variétés et ressources génétiques testées dans l'étude $\left(\mathrm{R}^{2}=0,91\right)$, y compris pour des populations dont la teneur en $\Delta^{9}$-THC n'est pas conforme aux standards communautaires $\left(\Delta^{9}\right.$-THC $>$ $0,20 \%)$.

Les deux critères étant équivalents, ils sont donc interchangeables, tout du moins lors de la mise en œuvre de PI. Sur la base de ces expérimentations et en tenant compte de la relation mentionnée pour les variétés autorisées (Figure 1), la valeur de $\alpha$ est de 0,19 . Par souci de simplification, nous proposons 0,20 comme valeur seuil du rapport $\alpha=\Delta^{9}-\mathrm{THC} / \mathrm{CBD}$.

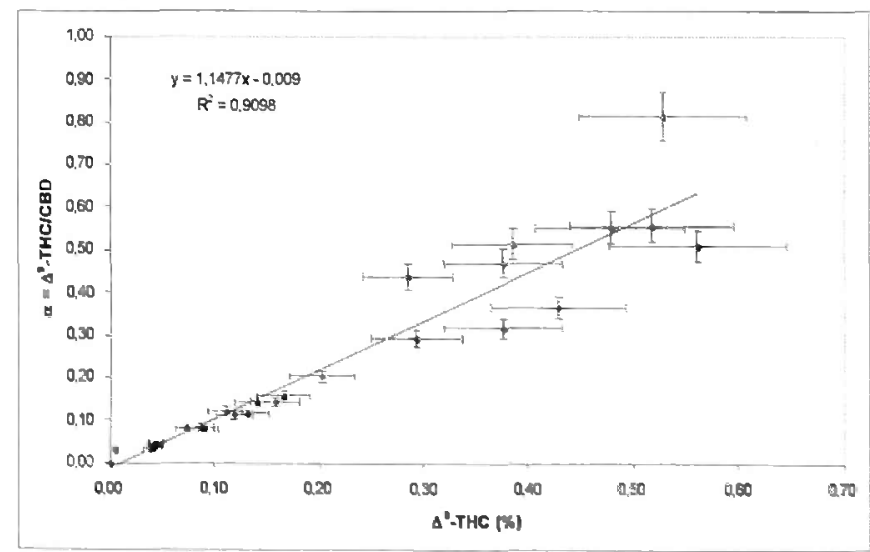

Figure 2 : Relation entre la seneur en $\Delta^{\circ}-T H C$ et la valeur du rapport $\alpha=\Delta^{2}$-THC / CBD (moyennes +/- écart-type, $n=20)$ dans les 28 variétés et ressources génériques de chanure issues de la collection FNPC. 
Rappelons que la difficulté logistique à mettre en œuvre cette procédure (analyse des tiers supérieurs de 500 plantes) est grande et que cela a conduit, en 2000, à la simplifier notamment dans le cadre des contrôles en routine des cultures de chanvre industriel. Mais cette simplification a entraîné des dérives dans les résultats moyens obtenus et un accroissement de leur variabilité (6).

L'utilisation du rapport $\alpha=\Delta^{\prime \prime}$-THC / CBD à la place de la détermination simple de la teneur en $\Delta^{9}$-THC n'a d'intérêt que si, lors de la simplification des protocoles pour réaliser les contrôles en routine, les biais observés précédemment sont levés (6). Il avait en effet été montré les influences que pouvaient avoir la partie prélevée, la date du prélèvement et le nombre de plantes sur la teneur en $\Delta^{9}$-THC. De telles influences se retrouvent-t-elles lors de la détermination du rapport $\alpha=\Delta^{9}$-THC / CBD ?

\section{Variabilité de la teneur en $\Delta^{9}$-THC et de celle du rap- port $\alpha=\Delta^{9}-\mathrm{THC} / \mathrm{CBD}$ Évaluation du rapport $\alpha=\Delta^{9}-T H C / C B D$ en fonction de la partie prélevée}

L'objectif est ici de savoir si les variations observées pour la teneur en $\Delta^{\prime}$-THC en fonction du type de prélèvement (feuilles, inflorescences) réalisé sur les plantes se retrouvent dans le rapport $\alpha=\Delta^{9}$-THC / CBD.

Ce test étant lourd à mettre en oeuvre, nous n'avons pu le réaliser sur les 13 variétés et 15 ressources génétiques précédentes. Aussi nous sommes nous focalisés sur 3 variétés : Fédora 17, Fédora 19, Félina 34, et trois populations issues de la collection : $\mathrm{P} 01, \mathrm{P} 02$ et $\mathrm{P} 03^{\prime}$. L'objectif restant de simplifier la procédure initiale de prélèvement, nous avons restreint à 100 le nombre de plantes dans l'échantillon.

\section{Méthodologie}

Cette expérimentation a été menée sur 100 plantes des 3 variétés et 3 populations retenues. Au stade fin floraison, on prélève sur chaque plante : une feuille sénescente (A), une feuille jeune (B), une grande feuille de l'inflorescence $(C)$, une petite feuille de l'inflorescence (D) et l'inflorescence sans feuille (E). Au sein de chaque variété ou population, et pour chacune des différentes parties, les 100 prélèvements sont mélangés. Le matériel végétal est alors analysé par chromatographie en phase gazeuse

' Le choix de ce matériel végétal a été guidé par la disponibilité des semences et par le souci de disposer de résultats couvrant la gamme la plus large possible : $\Delta^{\circ}-\mathrm{THC}=0,05$ à 0,13 pour F17, F19 et $\mathrm{F} 34$ et 0,29 à 0,53 pour $\mathrm{P} 01$ à $\mathrm{P} 03$. selon la technique habituelle. On détermine la teneur en $\Delta^{9}$-THC et le rapport $\alpha=\Delta^{9}$-THC / CBD.

De plus, pour chacune des variétés ou populations, un prélèvement selon la procédure initiale (PI) a également été réalisé.

Ce sont donc 36 analyses [( 3 variétés +3 populations) $x$ (5 parties différentes $+\mathrm{PI}$ )] qui ont donc été ici effectuées.

\section{Résultats et discussion}

Les résultats des dosages sont représentés sur les figures 3 et 4 . Il apparaît clairement (Figure 3) que le rapport $\alpha=\Delta^{9}$-THC / CBD est relativement constant quelle que soit la partie prélevée, et ce pour l'ensemble des 3 variétés $(F 17=0,05 \pm \mathbf{0 , 0 1} ; \mathrm{F} 34=0,09 \pm \mathbf{0 , 0 1}$; $\mathrm{F} 19=0,13 \pm \mathbf{0 , 0 1})$ et des 3 populations $(\mathrm{P} 01=0,26 \pm$ $\mathbf{0 , 0 2} ; \mathrm{P} 02=0,46 \pm \mathbf{0 , 0 4} ; \mathrm{P} 03=0,70 \pm \mathbf{0 , 0 6})$. De même, la valeur observée pour chacune des parties de la plante est similaire à celle obtenue lors d'un prélèvement selon PI.

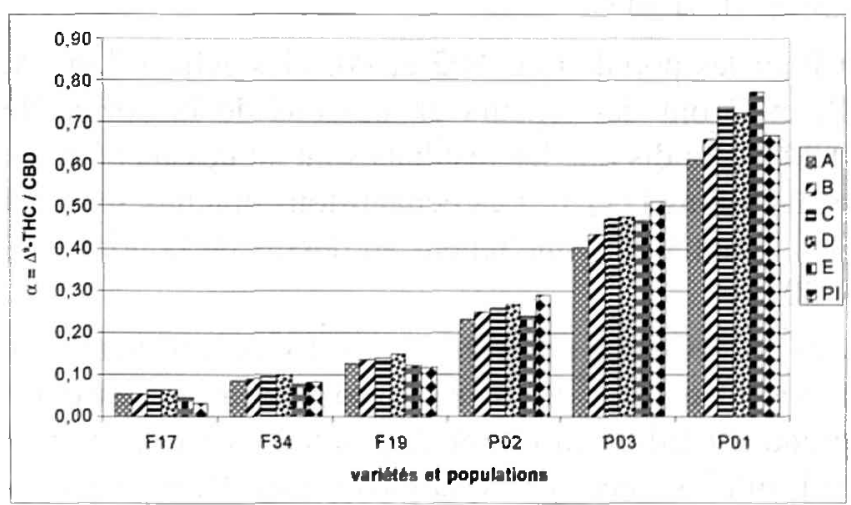

Figure 3 : Valeur du rapport $\alpha=\Delta^{9}-T H C / C B D$ en fonction du prélèvement réalisé Igrandes feuilles sénescentes (A), jeunes $(B)$ ou de l'inflorescence $(C)$; petites feuilles de l'inflorescence $(D)$; inflorescence sans feuille $(E)$; procédure initiale : $P I]$.

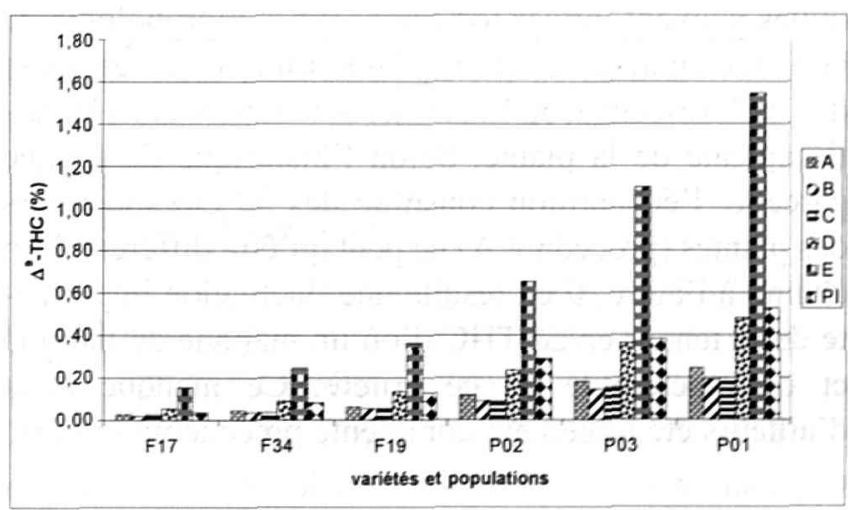

Figure 4 : Teneurs $\Delta^{9}-T H C$ en fonction du prélèvement réalisé /grandes feuilles sénescentes $(A)$, jeunes $(B)$ ou de l'inflorescence $(C)$; petites feuilles de l'inforescence $(D)$; inflorescence sans feuille (E) ; procédure initiale: $P[I$. 
La figure 4 montre que, pour les 3 variétés et les 3 populations, les feuilles (A, B et C), ont des teneurs moyennes en $\Delta^{9}$-THC comparables les unes aux autres, mais que cette teneur augmente dès lors que nous réalisons le prélèvement sur l'inflorescence (E) ou même sur une petite feuille de l'inflorescence (D).

Par ailleurs, en ce qui concerne le $\Delta^{9}$-THC, on peut constater que :

- Pour Fédora 17 (F17), les 5 échantillons A, B, C, D et $\mathrm{E}$ ont des teneurs en dessous de la norme de $0,20 \%$. Il en est de même pour l'échantillon moyen obtenu selon PI.

- Pour P01, les 5 échantillons A, B, C, D et E ont des teneurs au dessus de la norme de $0,20 \%$. Il en est de même pour l'échantillon moyen obtenu selon PI.

- Pour Fédora 19 (F19) et Félina 34 (F34), les 4 échantillons $\mathrm{A}, \mathrm{B}, \mathrm{C}$ et $\mathrm{D}$ ont des teneurs en dessous de la norme de $0,20 \%$, tandis que le résultat est au dessus pour l'échantillon E. L'échantillon obtenu par la procédure initiale a, quant à lui, une teneur en dessous de la norme de $0,20 \%$.

- Pour les populations P02 et P03, les échantillons A, $\mathrm{B}$, et $\mathrm{C}$ ont des teneurs en dessous de la norme de $0,20 \%$, tandis que les résultats sont au dessus pour les échantillons D et E. Les échantillons obtenus selon PI ont, quant à eux, une teneur au dessus de la norme de $0,20 \%$.

Ces observations relatives au $\Delta^{9}$-THC, confirment bien l'existence d'un gradient de concentration entre les grandes feuilles du bas de la plante (les moins riches) et l'inflorescence (partie la plus riche). Dans la procédure initiale, on ne cherchait pas à déterminer la teneur maximale mais, en prélevant le tiers supérieur, il était bien entendu tenu compte de ce gradient et donc des variations de taux existant entre les différentes parties vertes des plantes.

Dans les champs de production, les conditions climatiques peuvent influer fortement sur la morphologie de la plante en accentuant le volume foliaire au détriment de l'inflorescence, ou l'inverse, notamment dans la partie apicale de la plante. Selon l'historique de chaque parcelle, l'échantillon constitué des $30 \mathrm{~cm}$ supérieurs des plantes (procédure A) ne peut qu'être différent d'un champ à l'autre. Il en résulte une fluctuation importante de la teneur en $\Delta^{y}$-THC d'où un manque de fiabilité et de répétabilité de ce critère. Ce manque avait d'ailleurs été largement commenté précédemment (6).

Ce résultat confirme à nouveau le besoin de définir strictement une procédure d'échantillonnage précise tenant compte des difficultés rencontrées sur le terrain. Il avait été clairement montré que la procédure A ne donne pas un résultat fiable pour déterminer la teneur en $\Delta^{9}$-THC, en fonction des conditions culturales et climatiques annuelles (6). L'utilisation du rapport $\alpha=\Delta^{9}$ THC / CBD est au contraire fiable, même pour un prélèvement beaucoup plus petit, par exemple une feuille, ce qui réduit considérablement le volume de matière première à transporter et à traiter pour préparer l'échantillon à analyser.

\section{Évolution $d u$ rapport $\alpha=\Delta^{9}-T H C / C B D$ en fonction du stade de développement}

L'objectif est ici d'étudier l'évolution d'une part du $\Delta^{9}$ THC et d'autre part celle du rapport $\alpha=\Delta^{9}$-THC / CBD au cours du développement des plantes.

Une conclusion a déjà été apportée dans une précédente étude dans laquelle il avait été montré sur deux variétés (Fédora 17 et Secueni, voir ci-dessus) que le rapport $\alpha=\Delta^{9}$-THC / CBD reste constant quelle que soit la période où est effectué le prélèvement (19). Nous présentons ici de nouveaux résultats pour conforter ce travail antérieur. Dans un souci de simplification, ne sont présentés que les résultats de deux variétés : Fédora 17 et une ressource issue de la collection FNPC : P01.

\section{Méthodologie}

Cette expérimentation a été menée sur les 100 mêmes plantes de Fédora 17 et de P01 tout au long du cycle cultural. De mi-juin (semaine 25) à mi-septembre (semaine 37), on prélève sur chaque plante une feuille jeune (catégorie B du chapitre II-B), l'échantillonnage n'ayant pas pour but de déterminer la teneur en $\Delta^{9}$-THC des variétés mais de vérifier la stabilité du rapport $\alpha=$ $\Delta^{9}$-THC / CBD dans le temps. L'analyse a donc porté chaque semaine sur un échantillon constitué d'une feuille jeune de chacune des mêmes 100 plantes. Au stade fin floraison (semaine 32), on prélève également le tiers supérieur de ces 100 plantes de Fédora 17 et de P01 (ce qui ne détruit pas les plantes et permet de poursuivre l'expérimentation en continuant à prélever une feuille par plante chaque semaine). Il est ainsi possible de comparer ces valeurs à celle déterminée en appliquant la procédure initiale.

\section{Résultats et discussion}

L'évolution du rapport $\alpha=\Delta^{9}-\mathrm{THC} / \mathrm{CBD}$ au cours du développement des plantes, comparativement à la valeur de ce même rapport déterminé à partir d'un échantillon prélevé sur le tiers supérieur, est présentée sur la figure 5.

On observe que, pour une même variété, les variations sont très faibles, et les valeurs obtenues sont confondues tout au long du cycle avec la valeur de $\alpha=\Delta^{9}$ THC / CBD obtenue sur le prélèvement réalisé sur le tiers supérieur.

L'évolution de la teneur en $\Delta^{9}$-THC d'une feuille jeune 
au cours du développement des plantes, comparativement à la valeur déterminée à partir d'un échantillon prélevé sur le tiers supérieur, est présentée sur la figure 6. On observe des variations des teneurs en $\Delta^{9}$-THC dans les deux cas, et par ailleurs, la valeur obtenue lors du dosage du $\Delta^{9}$-THC sur le tiers supérieur est de 2 à 3 fois plus élevée que celle des feuilles.

En retenant l'évaluation du rapport $\alpha=\Delta^{9}$-THC / CBD, la conséquence de ces observations est la suivante : les prélèvements peuvent être réalisés à n'importe quel moment du cycle cultural.

De l'ensemble des expérimentations menées, il ressort que la mesure du rapport $\alpha=\Delta^{9}$-THC / CBD est très fortement corrélée $\left(R^{2}>0,96\right)$ à la seule mesure de la détermination du $\Delta^{y}$-THC dès lors que l'échantillonnage est effectué selon PI, à savoir le tiers supérieur de 500 plantes prélevées au stade fin floraison de la culture.

Les simplifications réalisées à partir de PI pour rendre logistiquement possibles les contrôles en routine des champs de production de chanvre (procédure A) ont entraîné des dérives des mesures, notamment en ne prélevant que $30 \mathrm{~cm}$ au lieu du tiers supérieur et en allongeant la période de prélèvement au lieu de prélever à un stade végétatif précis.

L'utilisation du rapport $\alpha=\Delta^{9}$-THC / CBD devrait permettre de s'affranchir des dérives constatées et d'aller encore plus loin dans la simplification en prélevant :

- une feuille par plante (d'où un volume très réduit et donc une manutention plus aisée et un gain de temps dans le traitement des échantillons) ;

- quasiment à tout moment du cycle cultural (d'où un étalement de la période des prélèvements). En prenant une feuille située hors de l'inflorescence, le prélèvement peut même être effectué avant la floraison.

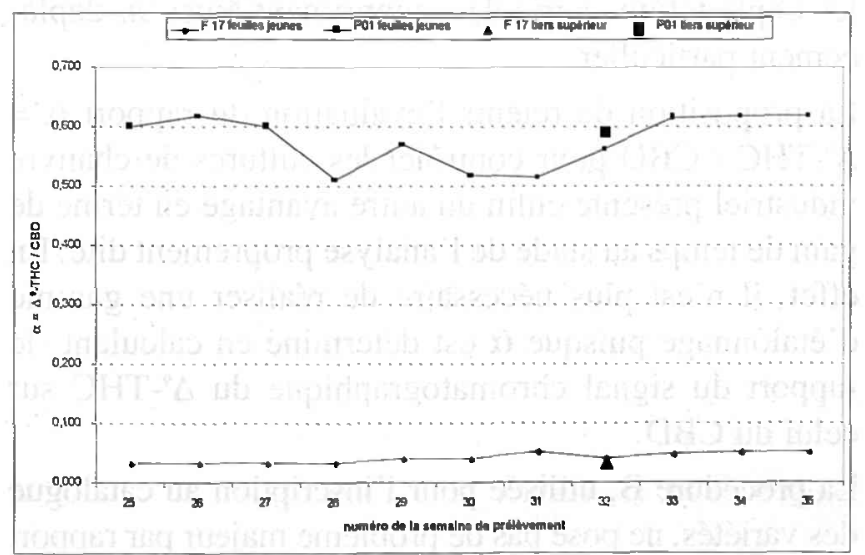

Figure 5 : Évolution de la valeur du rapport $\alpha=\Delta^{9}-T H C /$ $C B D$ dans les feuilles jeunes de deux variétés de chanvre (F 17 et POI) au cours du développement de la plante; comparaison avec la valeur de ce même rapport en prélevant le tiers supérieur des plantes.

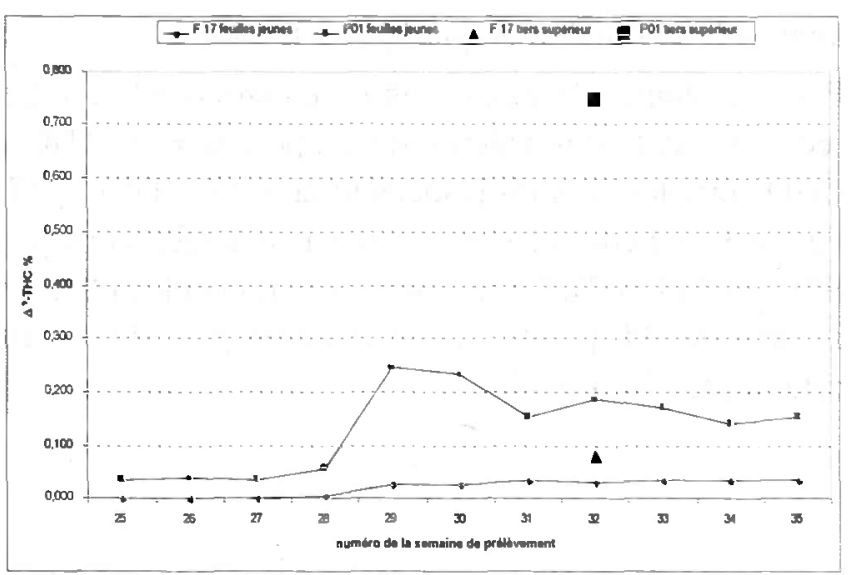

Figure 6 : Évolution de la teneur en $\Delta^{\circ}-T H C$ des feuilles jeunes de deux variétés de chanve ( F 17 et P01) au cours du développement de la plante; comparaison avec la teneur en $\Delta^{9}$-THC en prélevant le tiers supérieur des plantes.

\section{Détermination du nombre de plantes à prélever}

Il a donc été montré que, lorsque l'échantillon est constitué d'un nombre de plantes très important (500), le rapport $\alpha=\Delta^{9}$-THC / CBD peut remplacer la mesure seule du $\Delta^{9}$-THC. L'objectif ici est de voir l'impact d'un échantillonnage réduit sur ce rapport $\alpha=\Delta^{9}$-THC / CBD.

\section{Méthodologie}

Cette expérimentation a porté sur les 3 mêmes variétés : Fédora 17, Fédora 19 et Félina 34 et les 3 mêmes populations issues de la collection de la FNPC : P01, P02 et P03.

Les prélèvements ont été effectués dans les mêmes conditions, à savoir le tiers supérieur des plantes au stade fin floraison. Pour chaque variété et population, 5 tailles d'échantillons ont été testées : 50 - $100-150$ 200 ou 500 plantes. Pour chaque échantillonnage, 60 prélèvements ont été réalisés.

Ce sont donc 1800 analyses [ 3 variétés +3 populations) $x 5$ tailles d'échantillons $\times 60$ répétitions] qui ont été ici effectuées.

\section{Résultats et discussion}

Les moyennes (+/- écart type) des valeurs des rapports $\alpha=\Delta^{9}$-THC / CBD, fonction du nombre de plantes analysées sont présentés sur la figure 7 .

La figure 7 montre que, comme pour le $\Delta^{9}$-THC, l'échantillonnage (nombre de plantes dans l'échantillon) reste un facteur de variabilité très important (6).

Quel que soit le nombre de plantes dans l'échantillon, il existe toujours une imprécision dans la valeur moyenne obtenue. Cette imprécision diminue quand on 
augmente le nombre de plantes prélevées.

Pour 50 plantes, la figure 7 montre clairement certains recouvrements des valeurs du rapport $\alpha=\Delta^{9}$-THC / $\mathrm{CBD}$ entre les variétés respectant la norme (Fédora 17, Félina 34 et Fédora 19) et celles qui ne la respectent pas (P01, P02 et P03). Ce recouvrement n'est plus observé à partir de 100 plantes. Il est donc proposé de retenir cette taille d'échantillons.

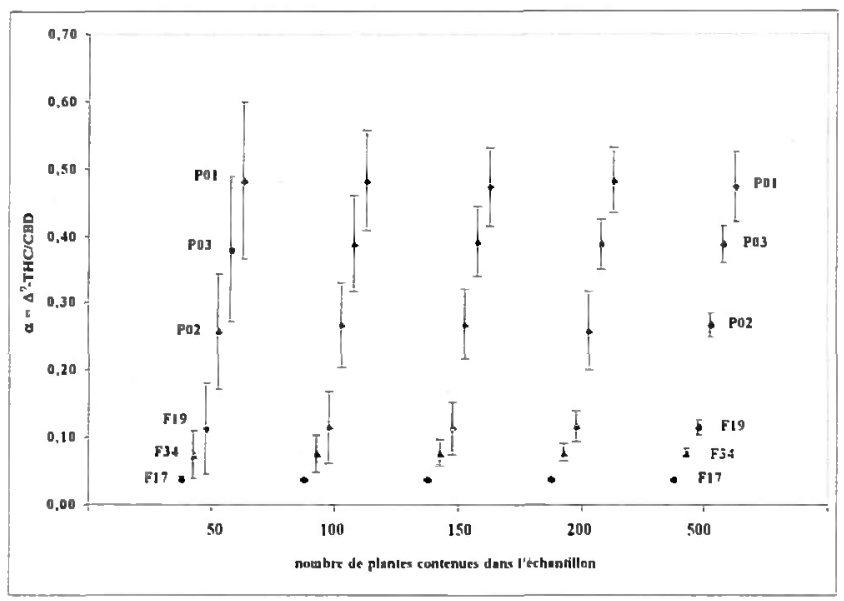

Figure 7 : Valeur du rapport $\triangle{ }^{9}$-THC / CBD (moyennes +/écart-type, $n=60$ ) de trois variétés de chanvre (Fédora 17 , Fédora 19, Félina 34) et de trois populations issues de la collection FNPC (POI, P02 et P03) en fonction de la taille de l'échantillon.

\section{Cas des variétés fibres de deuxième génération et fibres de troisième génération}

\section{Variétés fibres de deuxième génération}

Dans le cas de ce type de variétés (Santhica 27 et Santhica 70), il n'y a pas de $\Delta^{9}$-THC, le CBD est présent à l'état de trace, le cannabinoïde principal est le CBG (17).

Le rapport $\alpha=\Delta^{9}$-THC / CBD est donc nul, et cela est particulièrement visible sur la figure 1 (voir en bas à gauche). L'utilisation de ce critère peut donc être mise en ouvre pour le contrôle en routine des champs de production de ces variétés.

\section{Variétés fibres de troisième génération}

Ce type variétal sans cannabinoïdes a été décrit récemment (18), et aucune variété notoirement connue présentant cette caractéristique n'est à ce jour inscrite. L'absence de cannabinoïdes a été vérifiée dans toutes les parties de la plante et tout au long de son développement.
Pour ce chimiotype sans cannabinoïde, le rapport $\alpha=$ $\Delta^{9}$-THC / CBD ne peut donc pas être calculé mathématiquement.

Il y aura lieu dans ce cas de s'assurer que la teneur en $\Delta^{9}$-THC est inférieure au seuil de sensibilité des appareils de mesure.

\section{Conclusion}

Les différentes expérimentations ici effectuées et commentées permettent de montrer que la prise en considération du rapport $\alpha=\Delta^{\prime \prime}$-THC / CBD est un paramètre plus fiable que la seule mesure du $\Delta^{9}$-THC pour les contrôles en routine des champs de production de chanvre à fibres. On pourrait proposer une valeur maximale de 0,20 pour garantir que la teneur en $\Delta^{9}$-THC de la variété ne dépasse pas $0,20 \%$. En effet, ce rapport est beaucoup plus stable que la concentration en $\Delta^{9}$ THC quelles que soient l'époque du prélèvement et la partie prélevée. Cette plus grande stabilité permet de proposer que l'échantillon soit constitué d'une seule feuille prélevée sur chaque plante, au lieu des $30 \mathrm{~cm}$ supérieurs, d'où un volume très réduit qui permet d'envisager un nombre plus important de plantes prélevées, par exemple 100 au lieu de 50, gage d'une meilleure représentativité de la variété en culture. D'après cette étude, un échantillon constitué de 100 plantes permet de différencier les variétés respectant la norme $(\leq 0,20 \%)$ de celles qui ne la respectent pas.

Un autre avantage lié à la constance du rapport $\alpha=\Delta^{9}$ THC / CBD en fonction du développement des plantes est que le prélèvement peut être réalisé pendant un laps de temps plus important, indépendamment du stade végétatif de la culture, d'où une plus grande souplesse dans la programmation des travaux de prélèvements, voire de réaliser le prélèvement lors d'un des nombreux contrôles effectués sur les autres cultures dans les exploitations agricoles, supprimant ainsi un déplacement particulier.

La proposition de retenir l'évaluation du rapport $\alpha=$ $\Delta^{\prime \prime}$-THC / CBD pour contrôler les cultures de chanvre industriel présente enfin un autre avantage en terme de gain de temps au stade de l'analyse proprement dite. En effet, il n'est plus nécessaire de réaliser une gamme d'étalonnage puisque $\alpha$ est déterminé en calculant le rapport du signal chromatographique du $\Delta^{9}$-THC sur celui du CBD.

La procédure $\mathrm{B}$, utilisée pour l'inscription au catalogue des variétés, ne pose pas de problème majeur par rapport à la procédure initiale. Dans cette situation particulière de l'inscription, cette procédure, plus lourde à mettre en œuvre mais sur un nombre de cas plus restreint, doit être conservée pour garantir que la teneur en $\Delta^{\prime}$-THC de la variété ne dépasse pas la norme de $0,20 \%$. 


\section{Références}

1. Journal officiel des Communautés européennes Règlement (CE) $n^{\circ} 1164 / 89$ de la Commission du 28 avril 1989 relatif aux modalités concernant l'aide pour le lin textile et le chanvre.

2. Code de la Santé Publique - Arrêté du 22 août 1990 portant application de l'article R. 5181 J.O. du 4 octobre 1990.

3. Journal officiel des Communautés européennes Règlement $(\mathrm{CE}) \mathrm{n}^{\circ} 1177 / 2000$ de la Commission du 31 mai 2000 modifiant le règlement $(\mathrm{CE}) \mathrm{n}^{\circ} 1164 / 89$ relatif aux modalités d'aide concernant le lin textile et le chanvre.

4. Code de la Santé Publique - Arrêté du 24 février 2004 modifiant l'arrêté du 22 août 1990 portant application de l'article R. 5181 du code de la santé publique pour le cannabis. J.O. du 21 mars 2004.

5. Journal officiel des Communautés européennes Règlement (CE) $n^{\circ} 1672 / 2000$ de la Conseil du 27 juillet 2000 modifiant le règlement (CE) $n^{\circ} 1251 / 1999$ instituant un régime de soutien aux producteurs de certaines cultures arables, pour y inclure le lin et le chanvre destinés à la production de fibres.

6. Fournier G., Beherec O., Bertucelli S., Mathieu J.P. A propos des conditions d'échantillonnage pour le dosage du delta-9-tétrahydrocannabinol dans les variétés de chanvre à usage industriel. Ann. Toxicol. Anal. $2001 ; 13: 275-81$.

7. Turner C.E., Elsohly M.A., Boeren E.G. Constituents of Cannabis sativa L. XVII. A review of the neutral constituents. J. Nat. Prod. $1980 ; 43(2): 169-234$.

8. Turner C.E., Elsohly M.A. Constituents of Cannabis sati$v a \mathrm{~L}$. XVI. A possible decomposition of $\Delta$-9-tetrahydrocannabinol to cannabinol. J. Heterocycl Chem. 1979; 16 : 1667-8.

9. Fetterman P.S., Keith E.S., Waller C.W., Guerrero O., Doorenbos N.J., Quimby M.W. Mississippi-Grown Cannabis sativa L. : preliminary observation on chemical definition of phenotype and variations in tetrahydrocannabinol content versus age, sex, and plant part. J. Pharm. Sci. $1971 ; 60: 1246-9$.
10. Small E., Beckstead H.D. Cannabinoid Phenotypes in Cannabis sativa. Nature $1973 ; 245$ : 147-8.

11. Turner C.E., Elsohly M.A., Cheng P.C., Lewis G. Constituents of Cannabis sativa L., XIV : Intrinsic problems in classifying Cannabis based on a single cannabinoid analysis. J. Nat. Prod. $1979 ; 42: 317-9$.

12. Fournier G. Les chimiotypes du chanvre (Cannabis sativa L.). Intérêt pour un programme de sélection. Agronomie. $1981 ; 1: 679-88$.

13. Fournier G., Paris M. Détermination de chimiotypes à partir des cannabinoïdes chez le Chanvre à fibres monoïque (Cannabis sativa L.). Possibilités de sélection. Physiol. Vég. $1980 ; 18: 349-56$.

14. Debruyne D., Moulin M., Bigot M.C., Camsonne R. Identification and differentiation of resinous cannabis and textile cannabis : combined use of HPLC and high-resolution GLC. Bull. Narcotics, United Nations Publ. 1981 ; 33(2) : 49-58.

15. de Meijer E.P.M., van der Kamp H.J., Eeuwijk F.A. Characterisation of Cannabis accessions with regard to cannabinoid content in relation to other plant characters. Euphytica $1992 ; 62: 187-200$.

16. Giroud C. Analysis of cannabinoids in hemp plants. Chimia $2002 ; 56(3): 80-3$.

17. Fournier G., Béhérec O., Bertucelli S. Santhica 23 and 27 : deux variétés de chanvre (Cannabis sativa L.) sans 4-9-THC. Ann. Toxicol. Anal. $2004 ; 16: 128-32$.

18. Fournier G., Bausset J., Béhérec O., Bertucelli S. Une nouvelle souche de chanvre à fibres sans cannabinoödes. Ann. Toxicol. Anal. $2005 ; 17: 109-11$.

19. Fournier G., Béhérec O., Bertucelli S. Intérêt du rapport $\triangle$-9-THC/CBD dans le contrôle des cultures de chanvre industriel. Ann. Toxicol. Anal. $2003 ; 15: 250-9$. 